Pacific Journal of Mathematics

SOME INEQUALITIES FOR SYMMETRIC MEANS 


\title{
SOME INEQUALITIES FOR SYMMETRIC MEANS
}

\author{
P. S. Bullen
}

\section{This paper was received before the synoptic introduction became a requirement.}

1. In two recent papers, [3, 4], Everitt has generalised certain known inequalities, by replacing the known monotonicity of certain set (or sequence) functions by super-additivity; the sequence functions are zero if all the terms of the sequence are equal.

Included in the inequalities generalised is one due to Rado, [5, p. 61]. Bullen and Marcus, [1], recently proved a multiplicative analogue of this inequality and a generalisation to symmetric means. It is one of the intentions of this note to show that the corresponding sequence function, which is 1 when all the terms of the sequence are equal is logarithmically super-additive, (Corollary 5, below). Further properties of these sequence functions are then investigated.

2. $(a)=\left(a_{1}, \cdots, a_{m}\right)$ will denote an $m$-tple of positive numbers. $E_{r}(a), 1 \leqq r \leqq m$, is the $r$ th elementary symmetric function of $(a)$,

$$
E_{r}=E_{r}(a)=\sum \prod_{j=1}^{r} a_{i_{j}}, \quad E_{0}=1,
$$

the sum being over all $r$-tples, $i_{1}, \cdots, i_{r}$, such that $1 \leqq i_{1}<\cdots<i_{r} \leqq m$. $P_{r}(a)$ is the mean of $E_{r}(a)$,

$$
P_{r}=P_{r}(\alpha)=\left(\begin{array}{c}
m \\
r
\end{array}\right)^{-1} E_{r}
$$

If $m=n+q,(\bar{a})=\left(a_{1}, \cdots, a_{n}\right),(\widetilde{a})=\left(a_{n+1}, \cdots, a_{n+q}\right)$ and correspondingly $\bar{E}_{r}=E_{r}(\bar{a}), \quad \widetilde{E}_{r}=E_{r}(\widetilde{a})$, etc., if $r$ has suitable values. When $r=1$ the symmetric means are arithmetic means and will be written $P_{1}=A_{n+q}, \quad \bar{P}_{1}=\bar{A}_{n}, \quad \widetilde{P}_{1}=\widetilde{A}_{q}$. Similarly, $P_{n+q}, \bar{P}_{n}, \widetilde{P}_{q}$ are powers of geometric means and will be written $G_{n+q}^{n+q}, \bar{G}_{n}^{n}$ and $\widetilde{G}_{q}^{q}$ respectively.

3. It is known, $[5$, p. 52] that

(3) $s<t$ implies $P_{s}^{t} \geqq P_{t}^{s}$, with equality if and only if $a_{1}=\cdots=a_{m}$.

It is easily seen from (1) that

(i) if $s \leqq \min (n, q)$ then $E_{s}=\sum_{t=0}^{s} \bar{E}_{s-t} \widetilde{E}_{t}$,

(ii) if $s>\max (n, q)$ then $E_{s}=\sum_{t=0}^{n+q-s} \bar{E}_{n-t} \widetilde{E}_{s-n+t}$,

Received June 3, 1963. This research was supported by the U. S. Air Force Office of Scientific Research. 
(iii) if $q<s \leqq n$ then $E_{s}=\sum_{t=0}^{q} \bar{E}_{s-t} \widetilde{E}_{t}$.

Using these identities and (2) we have

LEMmA 1. (i) If $1 \leqq s \leqq n+q$

$$
P_{s}=\sum_{t=u}^{v} \lambda_{t}^{(s)} \bar{P}_{s-t} \widetilde{P}_{t},
$$

where $u=\max (s-n, 0), v=\min (s, q), \lambda_{0}^{(s)}=\lambda_{s}^{(s)}=1$ and $t /(), s$, $\lambda_{t}^{(s)}=\left[\left(\begin{array}{c}n \\ s-u-t\end{array}\right)\left(\begin{array}{c}q \\ u+t\end{array}\right)\right] /\left(\begin{array}{c}n+q \\ s\end{array}\right)$.

(ii) In particular if $a_{n+1}=\cdots=a_{n+q}=\beta$ then

$$
P_{s}=\sum_{t=u}^{v} \lambda_{t}^{(s)} \bar{P}_{s-t} \beta^{t},
$$

and if in addition $\alpha_{1}=\cdots=a_{n}=\alpha$,

$$
P_{s}=\sum_{t=u}^{v} \lambda_{t}^{(s)} \alpha^{s-t} \beta^{t} .
$$

When $q=1$ this reduces to formulae (2) and (4) of [1].

4. We are now in a position to state and prove

THEOREM 2. Let $1 \leqq r \leqq k \leqq n+q$ and $u=\max (r-n, 0), v=$ $\min (r, q), w=\max (k-n, 0), x=\min (k, q)$. Then

(i) if $v \leqq w$ and $r-u \leqq k-x$

$$
\frac{P_{r}^{k / r}}{P_{r}} \geqq \frac{\bar{P}_{r-u}^{(k-x) /(r-u)}}{\bar{P}_{k-x}} \frac{\widetilde{P}_{v}^{w / v}}{\widetilde{P}_{w}},
$$

(ii) if $v \leqq w$

$$
\frac{P_{r}^{k / r}}{P_{k}} \geqq \frac{\widetilde{P}_{v}^{w / v}}{\widetilde{P}_{w}},
$$

with equality in each case if and only if either $r=k$ or $a_{1}=\cdots=a_{n+q}$.

Before proceeding with the proof it should be noted that the condition $v \leqq w$ becomes $r-u \leqq k-x$ if $n$ and $q$ are interchanged. So if $r-u \leqq k-x$ inequality (8) holds, with the role of $n$ and $q$ interchanged; or equivalently $P_{r}^{k / r} / P_{k} \geqq \bar{P}_{r-u}^{(k-x) /(r-u)} / \bar{P}_{k-x}$. If neither $v \leqq w$ nor $r-u \leqq k-x$ then, from (3), nothing is true. The condition $v \leqq w$ is equivalent to $\min (r, q) \leqq \max (k-r, 0)$ and for this either $r<q$ and $k \geqq n+r$ or $r \geqq q$ and $k=n+q$; that is $k \geqq n+v$. For both $v \leqq w$ and $r-n \leqq k-x$ either $r<\min (n, q)$ and $k \geqq r+$ $\max (n, q)$ or $r \geqq \min (n, q)$ and $k=n+q$.

Proof of Theorem 2. If $r=k$ the results are trivial so assume $r<k$. Rewrite (7) as 


$$
L=\frac{P_{k}^{r}}{\bar{P}_{k-x}^{r} \widetilde{P}_{w}^{r}} \leqq \frac{P_{r}^{k}}{\bar{P}_{r-u}^{[(k-x) /(r-u)] r} \widetilde{P}_{v}^{w r / v}}=R
$$

By (4) with $s=r$

$$
P_{r}^{k}=\left(\sum_{t=u}^{v} \lambda_{t}^{(r)} \bar{P}_{r-t} \widetilde{P}_{t}\right)^{k}
$$

Using (3) on each term of this sum

$$
P_{r}^{k} \geqq\left(\sum_{t=u}^{v} \lambda_{t}^{(r)} \bar{P}_{r-u}^{(r-t) /(r-u)} \widetilde{P}_{v}^{t / v}\right)^{k} .
$$

By (6) the right hand side of this inequality is the $k$ th power of the $r$ th symmetric mean of $b_{1}, \cdots, b_{n+q}$ where $b_{1}=\cdots=b_{n}=P_{r-u}^{1 /(r-u)}$ and $b_{n+1}=\cdots=b_{n+q}=P_{v}^{1 / v}$. Using (3), (6) and $r<k$ this gives

$$
\begin{aligned}
P_{r}^{k} & \geqq\left(\sum_{t=w}^{x} \lambda_{t}^{(k)} \bar{P}_{r-u}^{(k-t) /(r-u)} \widetilde{P}^{t / v}\right)^{r} \\
& =\bar{P}_{r-u}^{[(k-x) /(r-u)] r} \widetilde{P}^{w r / v}\left(\sum_{t=w}^{x} \lambda_{t}^{(k)} \bar{P}_{r-u}^{(x-t) /(r-u)} \widetilde{P}_{v}^{(t-w) / v}\right)^{r} .
\end{aligned}
$$

On rewriting we get,

$$
R \geqq\left(\sum_{t=w}^{x} \lambda_{t}^{(k)} \bar{P}_{r-u}^{(x-t) /(r-u)} \widetilde{P}_{v}^{(t-w) / v}\right)^{r}=S, \quad \text { say . }
$$

Similarly by (4)

$$
P_{k}^{r}=\left(\sum_{t=w}^{x} \lambda_{t}^{(k)} \bar{P}_{k-t} \widetilde{P}_{t}\right)^{r}
$$

Using (3) on each term of this sum gives

$$
\begin{aligned}
P_{k}^{r} & \leqq\left(\sum_{t=w}^{x} \lambda_{t}^{(k)} \bar{P}_{k-x}^{(k-t) /(k-x)} \widetilde{P}_{w}^{t / w}\right)^{r} \\
& =\bar{P}_{k-x}^{r} P_{w}^{r}\left(\sum_{t=w}^{x} \lambda_{t}^{(k)} \bar{P}_{k-x}^{(x-t) /(r-x)} \widetilde{P}_{w}^{(t-w) / w}\right)^{r} .
\end{aligned}
$$

Rewriting we have that

$$
L \leqq\left(\sum_{t=w}^{x} \lambda_{t}^{(k)} \bar{P}_{k-x}^{(x-t) /(k-x)} \widetilde{P}_{w}^{(t-w) / w}\right)^{r}=T, \text { say . }
$$

By the condition in (i) and (3), $T \leqq S$, which proves (7). Some terms in the above proof become undefined in certain limiting cases. If they are defined to be 1 the proof is then correct. Finally, since $r<k$, the inequality is clearly strict when (3) is. This completes the proof of (i).

To prove (ii) the procedure is similar except that when (3) is applied to the right hand sides of (9) and (10) it is applied to the 
second part of each term only, that is to $\widetilde{P}_{t}$. The analysis is then the same with (5) being used instead of (6).

Corollary 3.

$$
\left(\frac{A_{n+q}}{G_{n+q}}\right)^{n+q} \geqq\left(\frac{\bar{A}_{n}}{\bar{G}_{n}}\right)^{n}\left(\frac{\widetilde{A}_{q}}{\widetilde{G}_{q}}\right)^{q}
$$

with equality if and only if $a_{1}=\cdots=a_{n+q}$.

Proof. From Theorem 2(i) with $r=1, k=n+q$.

COROLlaRY 4. If $1 \leqq r \leqq s \leqq n$ then

$$
\frac{P_{r}^{s+1}}{P_{s+1}^{r}} \geqq \frac{\bar{P}_{r}^{s}}{\bar{P}_{s}^{r}},
$$

and in particular

$$
\left(\frac{A_{n+1}}{G_{n+1}}\right)^{n+1} \geqq\left(\frac{\bar{A}_{n}}{\bar{G}_{n}}\right)^{n},
$$

with equality if and only if $a_{1}=\cdots=a_{n+1}$.

Proof. From Theorem 2 (ii) with $k=s+1, n=1$. These results are those in [1].

Finally if $r\{(a)\}=r(a)=\left(A_{m} / G_{m}\right)^{m}$ then we have

COROLlaRY 5. $\log r\{(\bar{a}) \cup(\widetilde{\alpha})\} \geqq \log r(\bar{a})+\log r(\widetilde{\alpha})$.

5. The above inequalities (11), (13) and that due to Rado, [5, p. 61] can be further generalised by the use of weighted means. Let $(w)=\left(w_{1}, \cdots, w_{n}\right)$ be an $m$-tple of nonnegative numbers, not all zero. Define

$$
\begin{gathered}
W_{r}=\sum_{i=1}^{n} w_{r}, \quad W_{r}>0, \\
A_{r}=A_{r}^{(w)}=\frac{1}{W_{r}} \sum_{i=1}^{r} w_{i} a_{i}, \quad G_{r}=G_{r}^{(w)}=\left(\prod_{i=1}^{r} a_{i}^{w_{i}}\right)^{1 / W_{r}} .
\end{gathered}
$$

It is known that

$$
G_{r} \leqq A_{r} \text {, with equality only when } a_{1}=\cdots=a_{n} .
$$

A generalisation of Rado's inequality and (13) is given by

THEOREM 6.

$$
W_{n}\left(A_{n}-G_{r}\right) \leqq W_{n+1}\left(A_{n+1}-G_{n+1}\right),
$$




$$
\left(\frac{A_{n}}{G_{n}}\right)^{W_{n}} \leqq\left(\frac{A_{n+1}}{G_{n+1}}\right)^{W_{n+1}},
$$

with equality if and only if $a_{1}=\cdots=a_{n+1}$.

Proof. The proofs are exactly those of the special cases. As direct proofs were not given in [1] they will be given here. In particular the proof of (15) is simpler than that suggested in [5].

(15) is equivalent to $G_{n+1} \leqq\left(W_{n} / W_{n+1}\right) G_{n}+\left(w_{n+1} / W_{n+1}\right) a_{n+1}=U$, say.

$$
\begin{aligned}
G_{n+1} & =G_{n}^{\left(W_{n} / W_{n+1}\right)} a_{n+1}^{\left(w_{n+1}\right) /\left(W_{n+1}\right)} \\
& \leqq U
\end{aligned}
$$

by an application of (14).

Similarly (16) is equivalent to

$$
A_{n+1} \geqq A_{n}^{\left(W_{n} / W_{n+1}\right)} a_{n+1}^{\left(w_{n+1}\right) /\left(W_{n+1}\right)}=V, \text { say }
$$

but

$$
\begin{aligned}
A_{n+1} & =\frac{W_{n}}{W_{n+1}} A_{n}+\frac{w_{n+1}}{W_{n+1}} a_{n+1} \\
& \geqq V,
\end{aligned}
$$

by an application of (14).

In a similar way we can prove

THEOREM 7.

$$
\begin{gathered}
W_{n+p}\left(A_{n+q}-G_{n+q}\right) \geqq \bar{W}_{n}\left(\bar{A}_{n}-\bar{G}_{n}\right)+\widetilde{W}_{q}\left(\widetilde{A}_{q}-\widetilde{G}_{q}\right), \\
\left(\frac{A_{n+q}}{G_{n+q}}\right)^{W_{n+q}} \geqq\left(\frac{\bar{A}_{n}}{\bar{G}_{n}}\right)^{\bar{W}_{n}}\left(\frac{\widetilde{A}_{q}}{\widetilde{G}_{q}}\right)^{\widetilde{W}_{q}},
\end{gathered}
$$

with equality if and only if $a_{1}=\cdots=a_{n+q}$.

Generalisations along the same lines are possible for the inequalities (7), (8) and (12). Suppose $(w a)=\left(w_{1} a_{1}, \cdots, w_{m} a_{m}\right)$; then define

$$
F_{r}(a)=\frac{E_{r}(w a)}{E_{r}(w)}
$$

a generalisation of $P_{r}(\alpha)$, to which it reduces if $w_{1}=\cdots=w_{m} \neq 0$. The two $m$-tples $(a),(w)$ will be said to be similarly ordered if for all $i, j, a_{i} \leqq a_{j}\left(a_{i} \geqq a_{j}\right)$ implies $w_{i} \leqq w_{j}\left(w_{i} \geqq w_{j}\right)$.

THEOREM 8. If $(a)$ and $(w)$ are similarly ordered then

(i) $s<t$ implies $F_{s}^{t}>F_{t}^{s}$, with equality if and only if $a_{1}=\cdots=a_{m}$.

(ii) inequalities (7), (8) and (12) hold, subject to the relevant conditions, with $P$ replaced by $F$. 
Proof. The proof of (i) is exactly that of (3), [5, p. 53]. Then the inequalities follow as before.

The requirement that $(\alpha)$ and $(w)$ be similarly ordered is essential as the following example shows. If $(a)=(1,1,2)$ and $(w)=(2,1,1)$ then $F_{1}<F_{2}^{1 / 2}$ but $F_{2}^{1 / 2}>F_{3}^{1 / 3}$. The extreme case $s=1, t=m$ of (i) is a weaker form of (14) since $F_{m}^{1 / m}$ is the unweighted geometric mean whereas $F_{1}$ is the weighted arithmetic mean with the larger numbers having the larger weights.

6. In recent papers Diananda, [2] and Kober [6], have investigated further properties of $A_{n}-G_{n}$. We will now prove multiplicative analogues of their results. Let $(w)=\left(w_{1}, \cdots, w_{n}\right), w_{i}>0, W_{n}=1$ and define

$$
\begin{aligned}
& R_{n}=R_{n}^{(w)}=\frac{A_{n}^{(w)}}{G_{n}^{(w)}}=\frac{A_{n}}{G_{n}} \\
& L_{n}=\prod_{i, j=1}^{n}\left(\frac{a_{i}^{1 / 2} a_{j}^{-1 / 2}+a_{i}^{-1 / 2} a_{j}^{1 / 2}}{2}\right), \\
& \Lambda_{n}=\prod_{i, j=1}^{n}\left(\frac{a_{i}^{1 / 2} a_{j}^{-1 / 2}+a_{i}^{-1 / 2} a_{j}^{1 / 2}}{2}\right)^{w_{i} w_{j}},
\end{aligned}
$$

$w=\min \left(w_{1}, \cdots, w_{n}\right), W=\max \left(w_{1}, \cdots, w_{n}\right)$.

\section{Theorem 9.}

$$
\begin{aligned}
& L_{n}^{w /(n-1)} \leqq R_{n} \leqq E_{n}^{W}, \\
& \Lambda_{n}^{1 /(1-w)} \leqq R_{n} \leqq \Lambda_{n}^{1 / w},
\end{aligned}
$$

with equality if and only if $a_{1}=\cdots=a_{n}$.

Proof. The proofs of (19) and (20) are similar so only that of (20) will be given. Writing $\alpha=1 /(1-w)$ the left hand inequality in (20) can be rewritten as

$$
G_{n}^{1-\alpha} \Pi_{n}^{\alpha} \leqq A_{n}
$$

where

$$
\Pi_{n}=\prod_{i, j=1}^{n}\left(\frac{a_{i}+a_{j}}{2}\right)^{w_{i} w_{j}}
$$

The left hand side of (21) is equal to

$$
\prod_{1 \leqq i<j \leqq n}\left(\frac{a_{i}+a_{j}}{2}\right)^{2 \alpha w_{i} w_{j}} \prod_{i=1}^{n} \alpha^{\left\{\alpha w_{i}^{2}+(1-\alpha) w_{i}\right\}} .
$$

Since $\alpha w_{i}^{2}+(1-\alpha) w_{i} \geqq 0$ and $\sum_{1 \leqq i<j \leqq n} 2 \alpha w_{i} w_{j}+\sum_{i=1}^{n}\left\{\alpha w_{i}^{2}+(1-\alpha) w_{i}\right\}=$ 
1, an application of (14) gives (21).

The proof of the right hand inequality in (20) is slightly longer. The proof is by induction on $n$ and the result is trivial when $n=1$. By rewriting, the inequality is equivalent to

$$
\beta_{n}(a)=\frac{A_{n}^{w} G_{n}^{1-w}}{\Pi_{n}} \leqq 1
$$

Using (17) and (18) it is easy to show that

$$
\beta_{n}(a)=\frac{\left\{\left(1-w_{n}\right) A_{n-1}+w_{n} a_{n}\right\}^{w} G_{n-1}^{\left(1-w_{n}\right)(1-w)} a_{n}^{w_{n}\left(1-w_{n}-w\right)}}{\Pi_{n-1} \cdot \prod_{\imath=1}^{n-1}\left(\frac{a_{i}+a_{n}}{2}\right)^{2 w_{i} w_{n}}} .
$$

In particular therefore, if $a_{1}=\cdots=a_{n-1}=\alpha$,

$$
\beta_{n}(\alpha)=\frac{\left\{\left(1-w_{n}\right) \alpha+w_{n} a_{n}\right\}^{w} \alpha^{\left(1-w_{n}\right)\left(w_{n}-w\right)} a_{n}^{\left(w_{n}\left(1-w_{n}-w\right)\right.}}{\left(\frac{a_{n}+\alpha}{2}\right)^{2 w_{n}\left(1-w_{n}\right)}} .
$$

Further if $v=\min \left(w_{1}, \cdots, w_{n-1}\right)$ then $v \geqq w$ and

$$
\beta_{n-1}\left(a_{1}, \cdots, a_{n-1}\right)=\frac{A_{n-1}^{v} G_{n-1}^{1-v}}{\Pi_{n-1}^{1 /\left(1-w_{n} !^{2}\right.}} .
$$

Now, since $1-w_{n}-w \geqq 0$ and $w+\left(1-w_{n}\right)(1-w)+w_{n}\left(1-w_{n}-w\right)=$ $2 w_{n}\left(1-w_{n}\right)$, an application of (14) to (23) demonstrates (22) in this special case.

If we now assume $\beta_{n-1} \leqq 1$ then

$$
\begin{aligned}
\beta_{n} \leqq \frac{\beta_{n}}{\beta_{n-1}^{\left(1-w_{n}\right)^{2}}} & =\frac{\left\{\left(1-w_{n}\right) A_{n-1}+w_{n} a_{n}\right\}^{w} G_{n-1}^{\left(1-w_{n}\right)(1-w)-\left(1-w_{n}\right)^{2}(1-v)} a_{n}^{w} n^{\left(1-w_{n}-w\right)}}{A_{n-1}^{v\left(1-w_{n}\right)^{2}} \prod_{i=1}^{n}\left(\frac{a_{i}+a_{n}}{2}\right)^{2 w_{i} w_{n}}} \\
& \leqq \frac{\left\{\left(1-w_{n}\right) A_{n-1}+w_{n} a_{n}\right\}^{w} A_{n-1}^{\left(1-w_{n}\right)\left(w_{n}-w\right)} a_{n}^{w} n^{\left(1-w_{n}-w\right)}}{\prod_{i=1}^{n-1}\left(\frac{a_{i}+a_{n}}{2}\right)^{2 w_{i} w_{n}}}
\end{aligned}
$$

using (14). Without any loss of generality we can assume that $a_{n}=$ $\max \left(a_{1}, \cdots, a_{n}\right)$, when in particular $a_{n} \geqq A_{n-1}$. Then

$$
\begin{aligned}
\beta_{n}(a) & \leqq \frac{\left\{\left(1-w_{n}\right) A_{n-1}+w_{n} a_{n}\right\}^{w} A_{n-1}^{\left(1-w_{n}\right)\left(w_{n}-w\right)} a_{n}^{w_{n}\left(1-w_{n}-w\right)}}{\left(\frac{a_{n}+A_{n-1}}{2}\right)^{2 w_{n}\left(1-w_{n}\right)}} \\
& \leqq 1,
\end{aligned}
$$

by the particular case (23) with $\alpha=A_{n-1}$.

The cases of equality are immediate.

It might be remarked that if $W^{\prime}$ is second largest and $w^{\prime}$ the 
second smallest of $(w)$ tnen

$$
1 \leqq L_{n}^{w w^{\prime}} \leqq \Lambda_{n} \leqq L_{n}^{W W^{\prime}} .
$$

It is possible to generalise Hölder's inequality using Theorem 9.

Theorem 10 . Let $a_{i j} \geqq 0(i=1, \cdots, m, j=1, \cdots, n)$ and

$$
\sum_{i=1}^{m} a_{i j}=s_{j}>0 \quad(j=1, \cdots, n) .
$$

Then

$$
D \prod_{\jmath=1}^{n} s_{j}^{w_{j}} \geqq \sum_{\imath=1}^{m} \prod_{\jmath=1}^{u} a_{i j}^{w_{j}} \geqq d \prod_{\jmath=1}^{n} s_{j}^{w_{j}}
$$

where

$$
\begin{aligned}
D & =\min (1, L, A) \\
d & =\max (l, \lambda)
\end{aligned}
$$

and

$$
\begin{gathered}
L=\max _{i} L_{n}^{-(w / n-1)}\left(a_{i 1}, \cdots, a_{\imath n}\right)=\max _{i} L_{n i}^{-(w / n-1)} \\
=\max _{i} \Lambda_{n}^{-(1 / 1-w)}\left(a_{i 1}, \cdots, a_{i n}\right)=\max _{i} \Lambda_{n}^{-(1 / 1-w)} \\
l=\min _{i} L_{n, i}^{-W V}, \\
\lambda=\min _{i} \Lambda_{n, i}^{-1 / w} .
\end{gathered}
$$

Proof. A simple modification of the usual proof $[5$, p. 23] using Theorem 9 instead of (14).

\section{REFERENCES}

1. P. Bullen and M. Marcus, Symmetric means and matrix inequalities, Proc. Amer. Math. Soc. 12 (1961), 286-290.

2. P. H. Diananda, On some inequalities of H. Kober, Proc. Camb. Phil. Soc. 59 (1963), 341-346.

3. W. N. Everitt, On the Hölder inequality, J. London Math. Soc. 36 (1961), 145-148. 4. - On an inequality for the generalised arithmetic and geometric means, Amer. Math. Monthly 70 (1963), 251-253.

5. Hardy, Littlewood and Polya, Inequalities, Cambridge, 1934.

6. H. Kober, On the arithmetic and geometric means and on Hölder's inequality, Proc. Amer. Math. Soc. 9 (1958), 452-459. 


\section{PACIFIC JOURNAL OF MATHEMATICS}

\section{EDITORS}

H. Samelson

Stanford University

Stanford, California

R. M. Blumenthal

University of Washington

Seattle, Washington 98105
J. Dugundu

University of Southern California Los Angeles, California 90007

*Richard Arens

University of California

Los Angeles, California 90024

\section{ASSOCIATE EDITORS}
E. F. BECKENBACH
B. H. NeumanN
F. WOLF
K. YOSIDA

\section{SUPPORTING INSTITUTIONS}

UNIVERSITY OF BRITISH COLUMBIA CALIFORNIA INSTITUTE OF TECHNOLOGY UNIVERSITY OF CALIFORNIA MONTANA STATE UNIVERSITY

UNIVERSITY OF NEVADA

NEW MEXICO STATE UNIVERSITY

OREGON STATE UNIVERSITY

UNIVERSITY OF OREGON

OSAKA UNIVERSITY

UNIVERSITY OF SOUTHERN CALIFORNIA
STANFORD UNIVERSITY

UNIVERSITY OF TOKYO

UNIVERSITY OF UTAH

WASHINGTON STATE UNIVERSITY

UNIVERSITY OF WASHINGTON

AMERICAN MATHEMATICAL SOCIETY CALIFORNIA RESEARCH CORPORATION SPACE TECHNOLOGY LABORATORIES NAVAL ORDNANCE TEST STATION 


\section{Pacific Journal of Mathematics}

\section{Vol. 15, No. $1 \quad$ September, 1965}

Donald Charles Benson, Unimodular solutions of infinite systems of linear

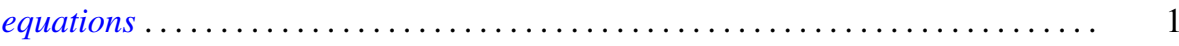

Richard Earl Block, Transitive groups of collineations on certain designs . . . . . . 13

Barry William Boehm, Existence of best rational Tchebycheff approximations .... . 19

Joseph Patrick Brannen, A note on Hausdorff's summation methods . . . . . . . . . . 29

Dennison Robert Brown, Topological semilattices on the two-cell ............ 35

Peter Southcott Bullen, Some inequalities for symmetric means . . . . . . . . . . 47

David Geoffrey Cantor, On arithmetic properties of coefficients of rational

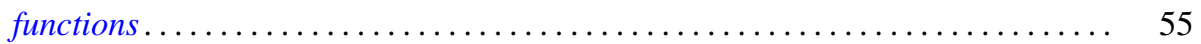

Luther Elic Claborn, Dedekind domains and rings of quotients . . . . . . . . . 59

Allan Clark, Homotopy commutativity and the Moore spectral sequence ........ 65

Allen Devinatz, The asymptotic nature of the solutions of certain linear systems of

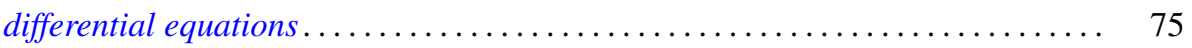

Robert E. Edwards, Approximation by convolutions ................... 85

Theodore William Gamelin, Decomposition theorems for Fredholm operators . . . . . 97

Edmond E. Granirer, On the invariant mean on topological semigroups and on

topological groups .................................. 107

Noel Justin Hicks, Closed vector fields . . . . . . . . . . . . . . . . . . . 141

Charles Ray Hobby and Ronald Pyke, Doubly stochastic operators obtained from

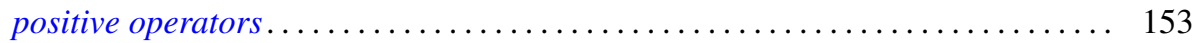

Robert Franklin Jolly, Concerning periodic subadditive functions . . . . . . . . . 159

Tosio Kato, Wave operators and unitary equivalence . . . . . . . . . . . . . . 171

Paul Katz and Ernst Gabor Straus, Infinite sums in algebraic structures . . . . . . . 181

Herbert Frederick Kreimer, Jr., On an extension of the Picard-Vessiot theory ...... 191

Radha Govinda Laha and Eugene Lukacs, On a linear form whose distribution is

identical with that of a monomial ......................... 207

Donald A. Ludwig, Singularities of superpositions of distributions . . . . . . . . . 215

Albert W. Marshall and Ingram Olkin, Norms and inequalities for condition

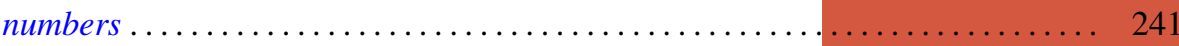

Horace Yomishi Mochizuki, Finitistic global dimension for rings . . . . . . . . . . 249

Robert Harvey Oehmke and Reuben Sandler, The collineation groups of division

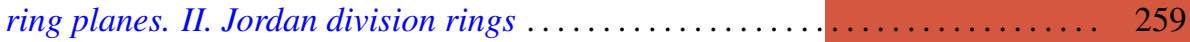

George H. Orland, On non-convex polyhedral surfaces in $E^{3} \ldots \ldots \ldots \ldots \ldots \ldots \ldots 267$

Theodore G. Ostrom, Collineation groups of semi-translation planes . . . . . . . . 273

Arthur Argyle Sagle, On anti-commutative algebras and general Lie triple

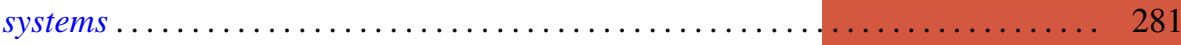

Laurent Siebenmann, A characterization of free projective planes . . . . . . . . . 293

Edward Silverman, Simple areas.................................. 299

James McLean Sloss, Chebyshev approximation to zero .................. 305

Robert S. Strichartz, Isometric isomorphisms of measure algebras . . . . . . . . . 315

Richard Joseph Turyn, Character sums and difference sets . . . . . . . . . . . . 319

L. E. Ward, Concerning Koch's theorem on the existence of arcs . . . . . . . . . . 347

Israel Zuckerman, A new measure of a partial differential field extension ......... 357 